\title{
Percutaneous Endoscopic Colostomy in a Patient with Chronic Intestinal Pseudo-Obstruction and Massive Dilation of the Colon
}

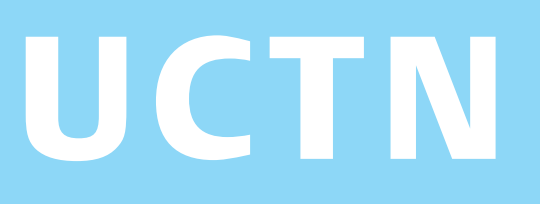

A 53-year-old woman with a 17-year history of multiple sclerosis, presented with abdominal pain and bowel distension. The computed tomography (CT) scan showed massive dilation of the colon (Figure $\mathbf{1}$ ). The patient had a long history of chronic intestinal pseudo-obstruction, which so far had always resolved after colonoscopic decompression and placement of a colon tube. This time once again, after endoscopic evacuation of the trapped air, the patient's situation improved rapidly. In the course of the hospital stay it became clear that decompression was needed every third day. Therefore, we aimed for a permanent solution, and we chose an endoscopic approach.

After bowel cleansing with standard polyethylene-glycol solution, a colonoscope was inserted into the proximal sigmoid. Transillumination was easily achieved, and a 14-Fr percutaneous endoscopic gastrostomy (PEG) set for enteral feeding (Nutricia Healthcare S.A., Fribourg, Switzerland) was used in a routine manner (Figure 2). Thereafter, the tube has been used for both decompression and irrigation. Since then, 6 months after the procedure, both her family doctor and her nurses agree that the patient has had much less abdominal discomfort and that the percutaneous endoscopic colostomy catheter is an easy-to-use device both for irrigation and decompression. Admittance to hospital for endoscopic decompression has not been necessary so far.

The use of PEG sets in the colon was first described in 2000, then used for fixation of a sigmoid volvulus [1]. The use of the catheter as an irrigation tube in children with chronic evacuation disorders has also been reported [2]. As demonstrated by our case, percutaneous endoscopic colostomy seems to be an easy method for permanent colonic decompression in patients with chronic intestinal pseudo-obstruction.

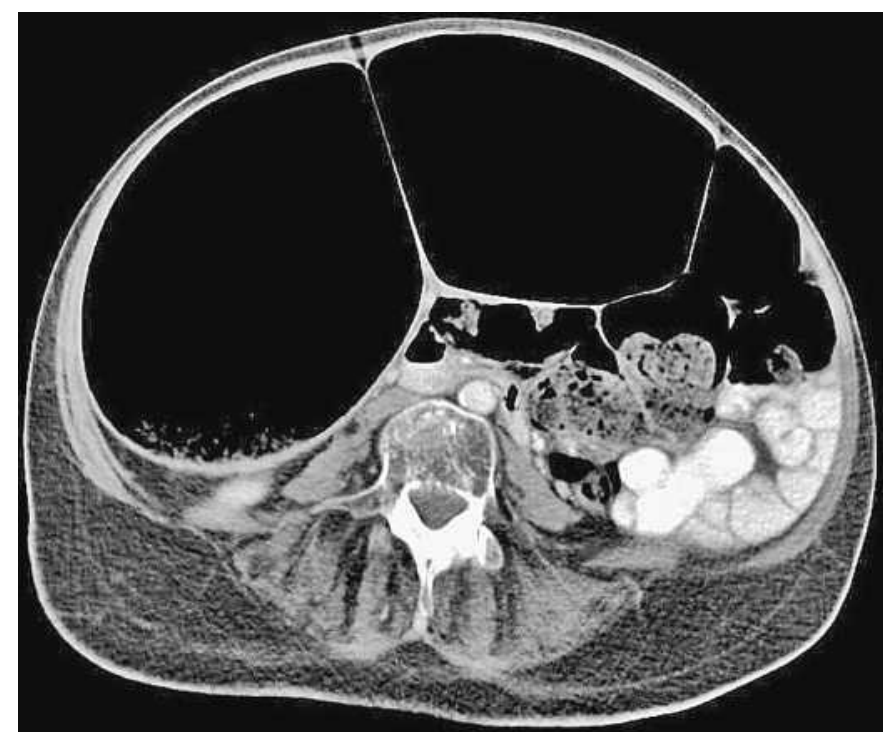

Figure 1 Computed tomography (CT) scan of the abdomen showing massive distension of the colon (maximum diameter was $18 \mathrm{~cm})$.

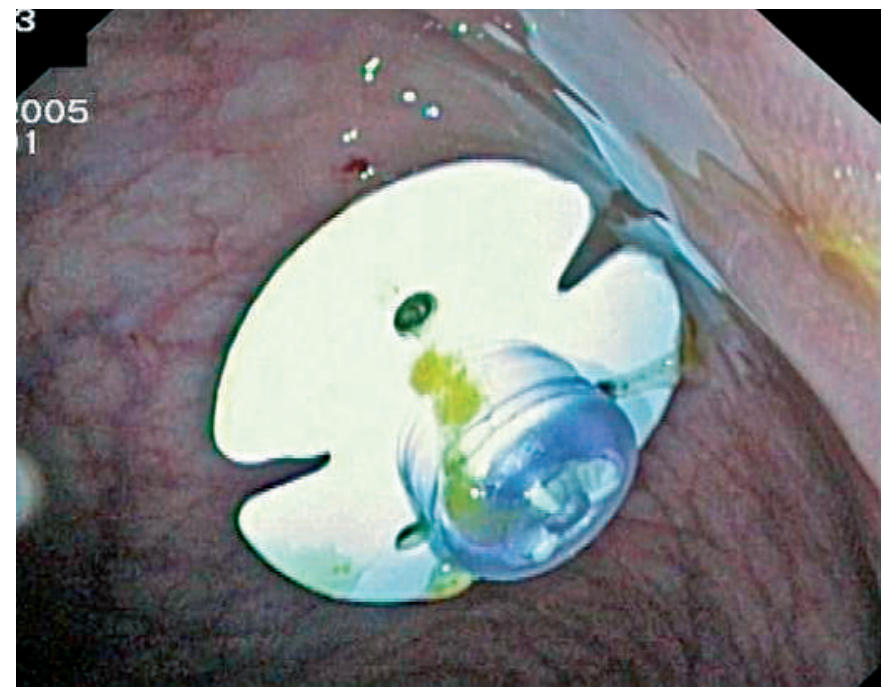

Figure 2 The 14-Fr standard percutaneous endoscopic gastrostomy (PEG) catheter placed in the sigmoid colon.

H. Einwächter ${ }^{1}$, P. Hellerhoff ${ }^{2}$, B. Neu ${ }^{1}$, C. Prinz ${ }^{1}$, R. Schmid ${ }^{1}$, A. Meining ${ }^{1}$

1 Department of Internal Medicine II, Klinikum Rechts der Isar, Technical University of Munich, Munich, Germany

2 Department of Radiology, Klinikum Rechts der Isar, Technical University of Munich, Munich, Germany.

\section{References}

${ }^{1}$ Daniels IR, Lamparelli MJ, Chave H, Simson JN. Recurrent sigmoid volvulus treated by percutaneous endoscopic colostomy. $\mathrm{Br} \mathrm{J}$ Surg 2000; 87: 1419

2 Rawat DJ, Haddad M, Geoghegan N et al. Percutaneous endoscopic colostomy of the left colon: a new technique for management of intractable constipation in children. Gastrointest Endosc 2004; 60: 39-43

\section{Corresponding Author}

\section{A. Meining, M.D.}

Department of Internal Medicine II, Klinikum Rechts der Isar, TU München 81675 Munich, Ismaninger Str. 22 Germany

Fax: $\quad+49-89-41404905$

E-mail: Alexander.Meining@lrz.tum.de

DOI: $10.1055 / \mathrm{s}-2006-925246$ 